\title{
Biomechanics Effects of Vibration Intervention on Achilles tendon in Rats among Different Frequencies
}

\author{
Fuming Gu \\ Chengdu Sport University, Chengdu, China \\ fuminggu@foxmail.com
}

Keywords: Biomechanics, structural mechanics, vibration, frequency, Achilles tendon.

\begin{abstract}
The objective of this objective was to investigate the biomechanical effects of vibration intervention training on tendon in rats. Sixty rats were randomly assigned to four groups: rest control group, $25 \mathrm{~Hz}$ vibration training group, $35 \mathrm{~Hz}$ vibration training group, and $45-\mathrm{Hz}$ vibration training group, 15 rats per group. Vibration training was provided to each training group of rats on the Power Plate vibration training platform. The conclusion showed that $25 \mathrm{~Hz}$ was the suitable frequency for vibration training of rat Achilles tendons. The training using suitable frequency can significantly improve the biomechanical property of the Achilles tendons and produce adaptive function changes so as to reduce the damage probability of the muscle-tendon complex and increased the exercise efficiency. In the meantime, the vibration frequency for the training shall not be the same as or close to the inherent frequency of the trained parts and systems to achieve the desired effect.
\end{abstract}

\section{Introduction}

In recent years, the vibration training was used in exercise training, rehabition, sports medicince, and so on., there some researchers have carried out some researches on the impact and role of vibration intervention. In these researches, taking some typical researches as follows, Karamanidis et al. examined whether an exercise-induced enhancement of the triceps surae and quadriceps femoris muscle-tendon unit capacities would affect mechanical and biological markers for knee osteoarthritis in the elderly. [1] Manal, et al. outlined a method for computing Achilles tendon moment arm using two reliable measurement instruments: ultrasound and video-based motion capture.[2] Maeda et al. investigated effects of ageing on biomechanical properties of healing tissues of the patellar tendon after the removal of its central portion. [3] Farris, assessed the effects of orthotic heel lifts on Achilles tendon force and strain during running.[4] Elvin et al. hypothesized that peak patellar tendon torques are greater in jump takeoff than in landing, and that there is a linear correlation between jump height and peak patellar tendon torque, Their findings showed that there was no significant difference in the peak patellar tendon torque experienced during takeoff and landing within individuals, a moderate correlation between maximum takeoff patellar tendon torques and jump height, a weak correlation between maximum landing patellar tendon torques and jump height, a moderate correlation between maximum $60 \%$ s isokinetic.[5] Elvin and Elvin, analyzed separately soleus and gastrocnemii reflexes to account for different effects of the vibrations on the reflex excitability of the slow soleus and of the gastrocnemii muscles.[6] Bojsen-Møller et al. investigated the relation between passive knee joint ROM and the mechanical properties of the patellar tendon.[7] Fukashiro et al. reviewed the research findings regarding the force and length changes of the muscle-tendon complex during dynamic human movements, especially those using ultrasonography and computer simulation.[8] In this study, the author investigated the effects of vibration training on the biomechanical effects on achilles tendom in rats among different frequencies. 


\section{Materials and Methods}

\subsection{Experimental Animals and Feeding Methods}

At the Experimental Animal Center under School of West China Medicine, Sichuan University, 60 healthy male SD rats with their weights ranging from $180 \mathrm{~g}$ to $210 \mathrm{~g}$ are nurtured and raised for 3 months in rack cages, with 5 rats per cage. The temperature for the movement and feeding environments is set between $18^{\circ} \mathrm{C}$ and $24^{\circ} \mathrm{C}$, and the relative humidity ranges from $45 \%$ to $55 \%$. They are exposed to natural light. In addition, all fed with standard pellet feed for rodents, and freely permitted to intake food and water.

\subsection{Scheme for Grouping and Training Animals}

After one week's adaptive feeding, the rats are randomly divided into the following four groups: the rest control group $(\mathrm{C})$, the $25 \mathrm{~Hz}$ vibration training group $(\mathrm{L})$, the $35 \mathrm{~Hz}$ vibration training group $(\mathrm{M})$, and the $45-\mathrm{Hz}$ vibration training group $(\mathrm{H})$, with 15 rats per group. Vibration training is provided to each training group of rats on the Power Plate vibration training platform. When being trained, 15 rats of the same group are simultaneously placed on the vibrating platform. Each rat is respectively deployed to self-made organic glassware where such rat is restrained from standing up, and such rat's lower limbs are subject to vibrating stimulation under gravity-resistant conditions. Each day requires 2 vibration training sessions with each training session spanning $15 \mathrm{~min}$. The interval between the two vibration training sessions is $5 \mathrm{~min}$. The vertical amplitude of each group is $3 \mathrm{~mm}$. Such training is required 5 days each week with 2 days for rest, and lasts for 8 weeks.

\subsection{Animal Sampling}

Sampling is conducted within $24 \mathrm{~h}$ following the final vibration training for each group of rats. The $10 \%$ chloral hydrate solution $(3 \mathrm{~mL} / \mathrm{kg})$ is used to anesthetize such rats via intraperitoneal injection. The rats' right Achilles tendon are sampled. Then, the extra peripheral tissues are cleaned away while leaving the tendons on both sides. The sample is wrapped in gauze, which has been soaked in a saline solution, and is immediately placed in a cryogenic vial pre-cooled with liquid nitrogen. After that, the vial is stored at $-80^{\circ} \mathrm{C}$ in a refrigerator for further testing.

\subsection{Mechanical Properties Testing}

At the Key Provincial Biomechanics Laboratory of Sichuan University, the thawed gastrocnemius muscles for testing are sandwiched in a test fixture and then placed on an electronic universal testing machine (AGIS-MS model) developed by Shimadzu. In a constant-temperature and constant-humid laboratory, a biomechanics test is run on such Achilles tendon. Before official testing, a preliminary calibration process for testing items is run. The preliminary calibration is run 10 times for cyclic tensile loading. The load ranges from 0 to $3 \mathrm{~N}$, and the loading rate is $3 \mathrm{~mm}$ per min. After the preliminary calibration test, an official tensile failure test with a loading rate of $3 \mathrm{~mm}$ per min is run. The successful experimental standard is that the tested items do not break off from their test fixture. Meanwhile, the load deformation curve is recorded. During the testing period, the needle fully injected with a saline solution is constantly dripped while maintaining its moisture. Thereafter, the TRAPEZIUM 2 software on the electronic universal testing machine processes the data and outputs structural and mechanical parameters (including the maximal load and the energy absorbed at the maximal load) and mechanical parameters of materials (including modulus of elasticity, maximal stress,).

\subsection{Data analysis}

All data collected from this study were analyzed with SPSS 16.0 for Windows. One way ANOVA, LSD multiple comparison were used in this study. All significant level was set at $\mathrm{P}<0.05$ level, and extremely significant level at $\mathrm{p}<0.01$. 


\section{Results}

\subsection{Characteristics of structural mechanics}

The one way ANVOA test of the structural mechanics parameters with LSD multiple comparison indicated the results as follows: (a) in the maximal load, it showed that there are extremely significant difference between groups $(\mathrm{P}<0.01)$. LSD multiple comparison showed that there were extremely significant difference between medium and control group, high frequency group and control group $(\mathrm{p}<0.01)$ (see fig 1.), and (b) in the energy absorbed at the maximal load, it showed that there were extremely significant difference between groups $(\mathrm{p}<0.01)$. LSD multiple comparison showed that there were extremely significant difference between medium and control group, high frequency group and control group $(\mathrm{p}<0.01)$ (see fig 2.).

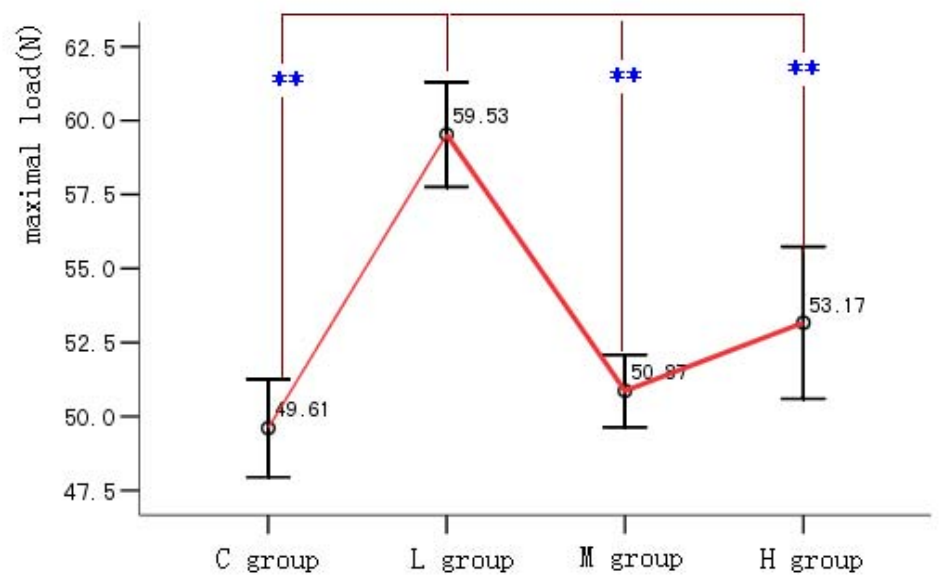

Figure 1. Comparison of characteristics of maximal loads between different vibration frequencies

Note: * indicates significant difference at $\mathrm{P}<0.05, * *$ indicates extremely significant difference at $\mathrm{P}<0.01$

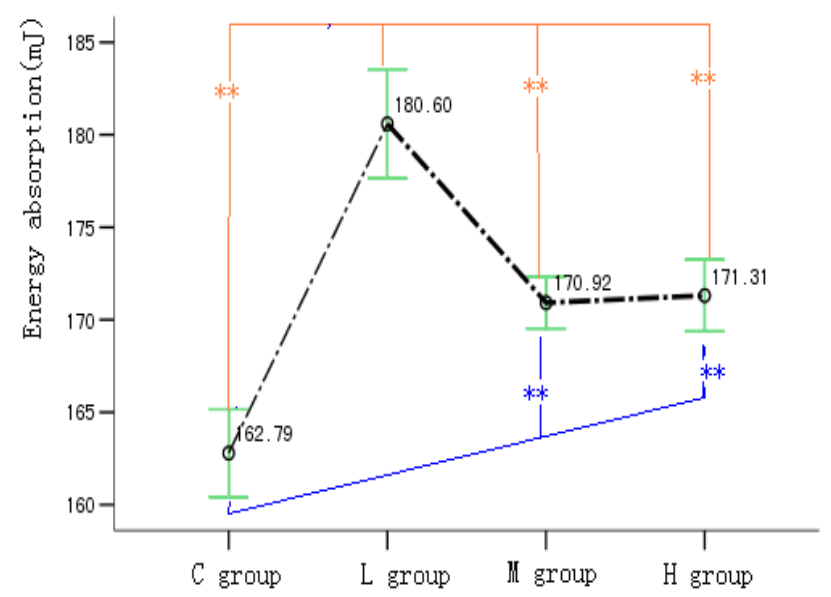

Figure 2. Comparison of energy absorbed at the maximal load between different vibration frequencies

\subsection{Characteristics of mechanics of material}

The one way ANVOA test of the mechanics of material parameters with LSD multiple comparisons indicated the results as follows: (a) in the modulus of elasticity ANVOA test, it showed that there were extremely significant differences between groups $(\mathrm{P}<0.01)$. LSD multiple comparison showed that there were extremely significant difference between medium and control group, high frequency group and control group ( $p<0.01$ ) (see fig 3.), (b) in the in the maximal stress ANVOA, it showed that there were extremely significant difference between groups $(p<0.01)$. LSD multiple comparison showed that there were extremely significant difference between medium and control group, high frequency group and control group $(\mathrm{p}<0.01)$ (see fig 4.), and (c) in the stress ANVOA test, 
it showed that there were extremely significant difference between groups $(p<0.01)$. LSD multiple comparison showed that there were extremely significant difference between medium and control group, high frequency group and control group $(\mathrm{p}<0.01)$ (see fig 5.).

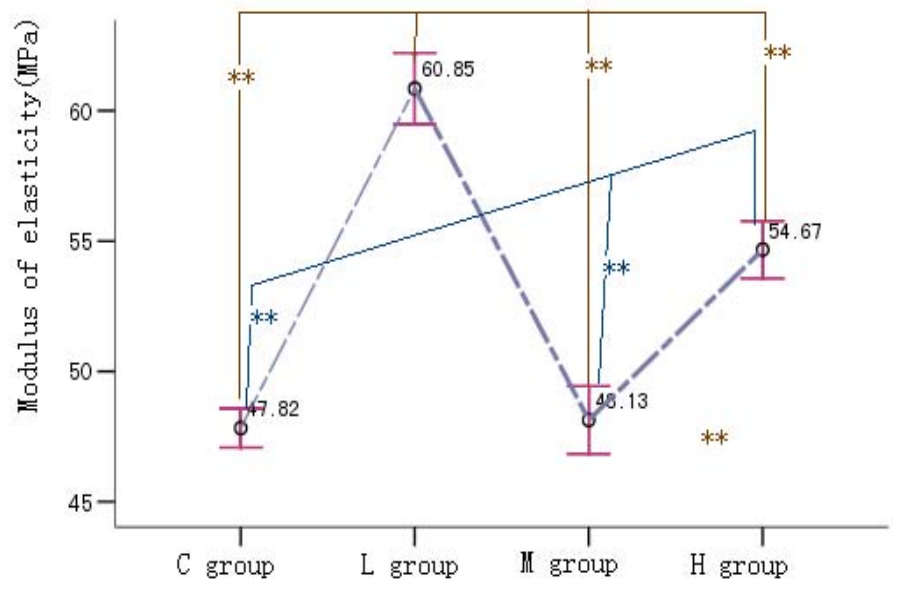

Figure 3. Comparison of modulus of elasticity between different vibration frequencies

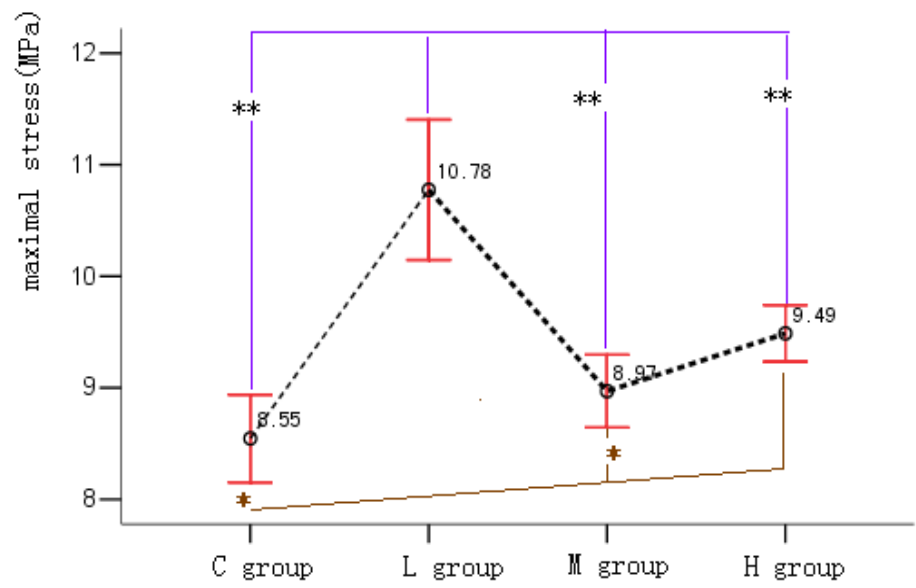

Figure 4. Comparison of maximal stress between different vibration frequencies

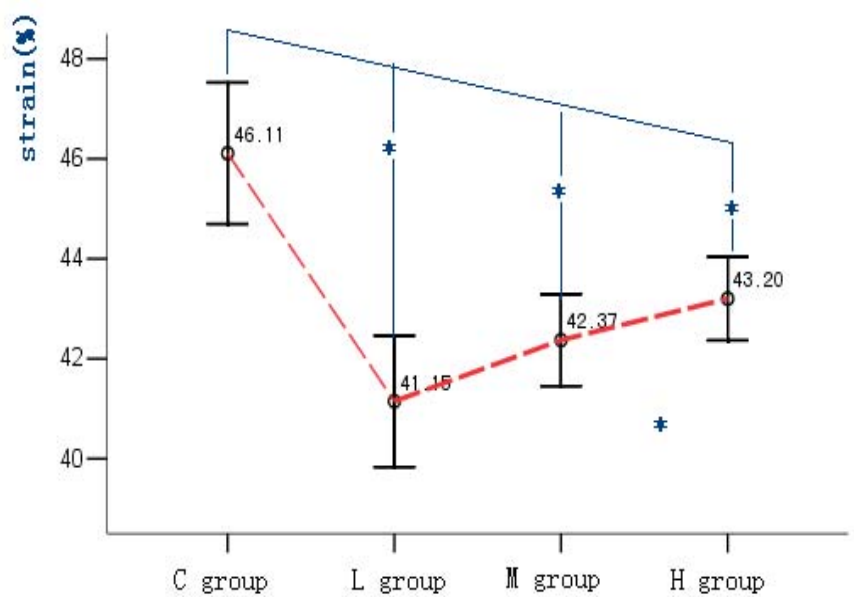

Figure 5. Comparison of stress between different vibration frequencies

\section{Analysis and Discussion}

\subsection{Influence of Vibration Training Using Different Frequencies on Mechanical Property of} Rat Tendon

Although there are many domestic and foreign researches on the influence of exercise training on mechanical property of tendons, the reported findings are quite different from each other due to the different types and ages of the experimental animals and the exercise modes and loads applied in the experiments. We have not located any reports on the influence of vibration training modes on 
mechanical property of rat tendons through the reference searching. Therefore, 3-month rats are selected for this research to exclude the influence of growth and development on the mechanical property of the tendon. The experiment suggests that the mechanical parameters of the tendons for the low-frequency $(25 \mathrm{~Hz})$ group and the high-frequency $(45 \mathrm{~Hz})$ group, such as maximum load, energy absorption, elasticity modulus and maximum stress, are significantly higher than those of the sedentary control group. However, the training results of the medium-frequency $(35 \mathrm{~Hz})$ group are not significant. The cause might be that $35 \mathrm{~Hz}$ vibration frequency is equal or very close to the inherent frequency of the lower limbs of the rats to trigger the limb resonance, causing overload that exceeds the allowable load limit of the tendon collagen fibers on the lower limbs of the rats that are forced to vibrating and produce slight damage on the fibers that will be repaired during the break of the vibration training, which means the fibers are repeatedly damaged and repaired during the whole vibration training cycle, causing no significant change on the biomechanical property of the rat tendons in $35 \mathrm{~Hz}$ training group.

According to the vibration theory, the acceleration amplitude of simple harmonic vibration is proportional to the square of vibration frequency; thus, the stress amplitude of the simple harmonic vibrated particle is also proportional to the square of vibration frequency. The biological tissues of the trained animals are forced to vibration in the vibration training. The higher the vibration frequency is, the higher the load born on the tissues is, which is proportional to the square of vibration frequency. Therefore the load on the tendon in the $45 \mathrm{~Hz}$ vibration training is much higher than that in the $25 \mathrm{~Hz}$ vibration training. It can be seen from Table 1 that the maximum load and elasticity modulus $(\mathrm{P}<0.01)$ and the maximum stress $(\mathrm{P}<0.05)$ of the low-frequency group are significant higher than those of the high-frequency group, which indicates that the improvement of mechanical property of rat tendons in the $25 \mathrm{~Hz}$ vibration training is better than that in the $45 \mathrm{~Hz}$ vibration training. Therefore, $25 \mathrm{~Hz}$ is a frequency more suitable to the vibration training of the rat tendons.

\subsection{Influence of Vibration Training on the Functional Adaptation of Rat Tendons}

Compared with the control group, the maximum load and stress and the elasticity modulus of the $25 \mathrm{~Hz}$ and $45 \mathrm{~Hz}$ groups are significantly increased and the strain under the maximum load is significantly reduced. However, because the $35 \mathrm{~Hz}$ vibration training that may cause resonance applies overload on the tendon, there is no significant change on the biomechanical parameters, which suggests that there is a limit for the influence caused by the increasing loads on the adaptability of the tendon and the adaptability will be stopped or turned to be harmful when the limit is exceeded.

The significant differences of biomechanical parameters such as maximum load and stress and elasticity modulus between the $25 \mathrm{~Hz}$ and $45 \mathrm{~Hz}$ groups indicate that the functional adaptability of the rat tendons to the loads are different within certain load limits and the adaptability will be better under appropriate load. Therefore, there is a best load value corresponding to the adaptability.

As other living tissues, the morphology and structure tendon tissues will change to adapt to the load they are born. However, there is a load limit and a best load for the tendon function adaptability. The load within the limit will improve the biomechanical property of the tendon significantly while that exceeds the limit will not produce function adaptability in the tendon, and massively excessive load will make the tendon impaired.

\subsection{Significance of the Mechanical Property Change of Rat Tendons after Training}

After the vibration training using appropriate frequency, the maximum load, stress and elasticity modulus of the rat tendon will be significantly increased while the strain under the maximum load will be significantly decreased. The increasing of maximum load can improve the ability of the tendon to bear the maximum load during the exercise, the increasing of maximum stress can improve the tendon strength, and the increasing of elasticity modulus will increase the tendon elasticity under certain deformation, which means stronger muscle force transmitted by the tendon under the same circumstance. Because part of or the whole collagen fibers in the tendon are damaged under certain strain to cause damages and ruptures of the tendon, the increasing of elasticity modulus and the decreasing of strain will reduce the possibility of tendon damages under stress.

Tendons not only transfer the muscle-produced force to the bones but also store elastic energy in the muscle concession contraction. The energy absorption of rat tendons under the maximum load is 
significantly increased, which means the capacity of elastic energy stored in the tendons is significantly increased. Based on the biomechanics, the energy storage and release of the tendon can increase the strength of the end in the muscle concentric contraction. Thus, the increasing of energy absorption can save the consumption and increase the economy of movements.

The improvement of the biomechanical property of the rat tendons after vibration training can improve the economy of the body movement and the safety coefficient of tendon work, and reduce the probability of tendon impairment during the high-intensity exercises.

\section{Conclusions}

$25 \mathrm{~Hz}$ is the suitable frequency for vibration training of rat tendons. The training using suitable frequency can significantly improve the biomechanical property of the rat tendons and produce adaptive function changes so as to reduce the damage probability of the muscle-tendon complex and increase the exercise efficiency. In the meantime, the vibration frequency for the training shall not be the same as or close to the inherent frequency of the trained parts and systems to achieve the desired effect.

\section{Acknowledgements}

This study was supported by the Sports Medicine key laboratory of General Administration of Sport of China / Sports Medicine key laboratory of Sichuan province Foundation (No: 2014 CTYY006) and National Natural Science Foundation of China (No: 11272068).

\section{References}

[1] Karamanidis, Kiros; Oberländer, Kai Daniel; Niehoff, Anja; Epro, Gaspar; Brüggemann, Gert-Peter. Effect of Exercise-Induced Enhancement of the Leg-Extensor Muscle-Tendon Unit Capacities on Ambulatory Mechanicsand Knee Osteoarthritis Markers in the Elderly.PLoS ONE. Jun2014, Vol. 9 Issue 6, p1-10.

[2] Manal, Kurt; Cowder, Justin D.; Buchanan, Thomas S., A Hybrid Method for Computing Achilles Tendon Moment Arm Using Ultrasound and Motion Analysis. Journal of Applied Biomechanics May2010, Vol. 26 Issue 2, p224.

[3] Maeda, Eijiro; Noguchi, Hitoshi; Tohyama, Harukazu; Yasuda, Kazunori; Hayashi, Kozaburo. Biomechanical study of healing of patellar tendon after resection of the central one-third in an adult-mature rabbit model.Bio-Medical Materials \& Engineering. 2013, Vol. 23 Issue 3, p173-181.

[4] Farris, Dominic James; Buckeridge, Erica; Trewartha, Grant; McGuigan, Miranda Polly.The Effects of Orthotic Heel Lifts on Achilles tendon Force and Strain during Running. Journal of Applied Biomechanics Nov2012, Vol. 28 Issue 5, p511.

[5] Elvin, Niell; Elvin, Alex; Scheffer, Cornie; Arnoczky, Steven; Dillon, Edwin; Erasmus, P. J., A Preliminary Study of Patellar Tendon Torques During Jumping.Journal of Applied Biomechanics Nov2009, Vol. 25 Issue 4, p360.

[6] Lapole, Thomas; Pérot, Chantal. Hoffmann reflex is increased after 14 days of daily repeated Achilles tendon vibration for the soleus but not for the gastrocnemii muscles. Applied Physiology, Nutrition \& Metabolism. Feb2012, Vol. 37 Issue 1, p14-20.

[7] Bojsen-Møller, J.; Brogaard, K.; Have, M. J.; Stryger, H. P.; Kjaer, M.; Aagaard, P.; Magnusson, S. P., Passive knee joint range of motion is unrelated to the mechanical properties of the patellar tendon.Scandinavian Journal of Medicine \& Science in Sports Aug2007, Vol. 17 Issue 4, p415. 
[8] Fukashiro, Senshi; Hay, Dean C.; Nagano, Akinori. Biomechanical Behavior of Muscle-Tendon Complex during Dynamic Human Movements.Journal of Applied Biomechanics May2006, Vol. 22 Issue 2, p131. 